\title{
Pancreatic cancer patients and thoracic surgeons: Could/should their clinic templates ever overlap?
}

\author{
Garrett L. Walsh, MD
}

\footnotetext{
From the Department of Thoracic and Cardiovascular Surgery, The University of Texas M.D. Anderson Cance Center, Houston, Tex.

Disclosures: Author has nothing to disclose with regard to commercial support.

Received for publication March 14, 2016; accepted for publication March 15, 2016; available ahead of print April $19,2016$.

Address for reprints: Garrett L. Walsh, MD, Department of Thoracic and Cardiovascular Surgery, The University of Texas M.D. Anderson Cancer Center, 1515 Holcombe Blvd, Unit 1489, Houston, TX 77030 (E-mail: gwalsh@mdanderson.org).

J Thorac Cardiovasc Surg 2016;152:83-4

$0022-5223 / \$ 36.00$

Copyright (C) 2016 Published by Elsevier Inc. on behalf of The American Association for Thoracic Surgery

http://dx.doi.org/10.1016/j.jtcvs.2016.03.048
}

A patient presented to the clinic at 9 months after an uneventful esophagectomy. Despite receiving neoadjuvant chemotherapy and radiation therapy and an encouraging final pathology of ypT0ypN0, he now demonstrates widely disseminated disease on routine reimaging. We ask ourselves, could there be any malignancy worse for a patient than esophageal cancer? And the answer is yes: pancreatic cancer. Although we have made advances through the use of trimodality approaches in our patients with esophageal cancer, our surgical oncology colleagues still struggle with a disease that often has an insidious onset and presents at an advanced, incurable stage in $80 \%$ of patients. In the remaining $20 \%$ of patients whose disease is localized and resectable, less than $30 \%$ can be cured. The 5-year overall survival is a dismal $6 \%$ for patients with this diagnosis.

Despite these grim statistics, Robinson and coauthors ${ }^{2}$ at the Moffitt Cancer Center were able to identify 25 patients with localized adenocarcinoma of the pancreas and 4 patients with biliary malignancies over a 20-year period $(<2 \%$ of their resected patients) who presented with synchronous or metachronous pulmonary lesions and underwent thoracic resection. Final pathology showed a metastatic lesion in 16 of the patients $(55 \%)$, primary lung cancer in 12 patients $(41 \%)$, and a benign lesion in 1 patient $(3 \%)$. The overall median survival was 28 months after pulmonary metastatectomy, with an estimated 5-year survival of $37 \%$, which is comparable to the survival of other solid organ metastases that are typically resected from the lungs (eg, colon, sarcoma, breast). The nonmetastatic group (lung cancer and benign) had a 78-month median survival. There were no surgical deaths, and morbidity was minimal.

I was intrigued by the authors' results and went back and reviewed our prospective database at the M.D. Anderson Cancer Center, and found very similar results. Over the past 17 years (1998-2015), we have performed pulmonary metastasectomy in 3128 patients. Twenty-five patients had

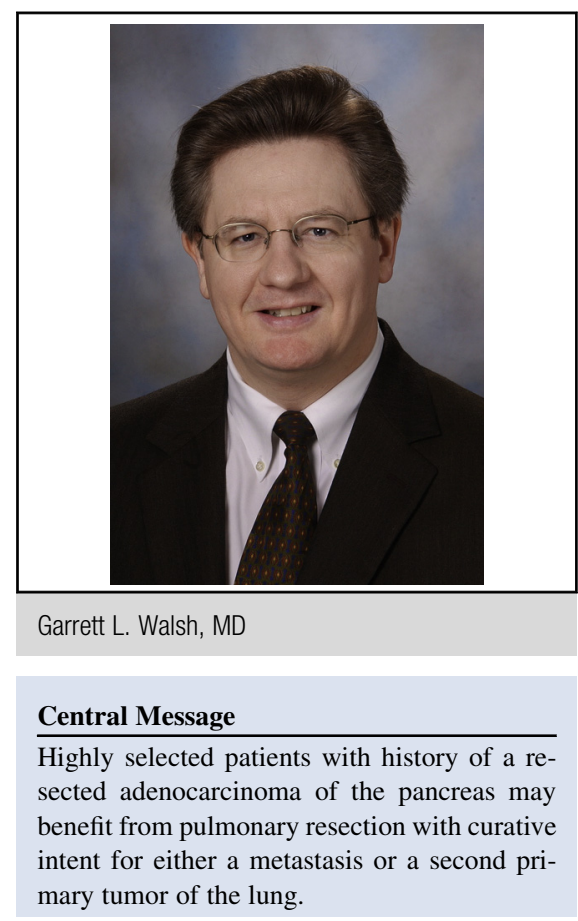

See Article page 75 . a previous pancreatic/biliary malignancy $(8 \%$ of our metastatasectomy cases and approximately $2 \%$ of our resected pancreas cases). Final pathology demonstrated metastatic disease in $15(60 \%)$, lung cancer in $9(36 \%)$, and a benign histology in $1(4 \%)$.

A few points come to light that should be emphasized:

1. Our oncology colleagues are generally very good at screening and selecting patients who may benefit from a thoracic surgical referral. Although rare, there are patients with a clearly different biology, with recurrence in the lung only and not in the typical locations of the pancreatic bed, liver, and abdominal cavity, who might benefit from surgical resection. ${ }^{3}$ Some colleagues, however, would also argue that there is a paucity of data suggesting that any pulmonary metastasectomy, regardless of histology, does little to alter the long-term survival of patients. We are merely selecting those with a favorable biology who may have a long survival even without surgery. In fact, this may even apply to this rare subset of pancreatic patients with documented stage IV disease.

2. An assumption that a patient has stage IV disease based on a pulmonary lesion may be inaccurate, given that 
benign lesions do occur. Indeterminate pulmonary nodules are frequently identified at the time of the initial diagnosis. ${ }^{4}$ Tissue confirmation by either transthoracic needle or video-assisted thoracocscopic surgery resection before diagnosing stage IV disease in a patient with a potential locally resectable lesion is warranted.

3. Smoking is a risk factor for the development of pancreatic cancer and lung cancer. As a result, nearly $40 \%$ of the pulmonary lesions are in fact lung cancers that may be identified at an early stage because of the close radiologic surveillance that pancreatic patients receive. Synchronous, resectable primary lesions are also possible.

Despite the low mortality and morbidity of pulmonary resections, we would weigh several factors before proceeding with a resection. For a patient with a diseasefree interval of $<1$ year who develops a pulmonary nodule or has an indeterminate pulmonary nodule identified on presentation that begins to grow, we often would proceed first to a needle biopsy to see whether histology can be obtained. If metastatic disease is confirmed, we would consider chemotherapy and follow the lesion for several months to ensure that widespread metastatic disease does not blossom. ${ }^{5}$ Some $80 \%$ of metastatic disease generally occurs within the first 2 years of the initial diagnosis. If only the nodule progresses and no other lesions develop, then we would proceed to resection. If a lung cancer is discovered, then the patient could be offered resection or considered for stereotactic body radiation therapy if deemed at high risk for developing metastatic disease based on the pathology of the resected primary tumor. ${ }^{6}$ The extent of pulmonary resection must be tempered to the patient's risk factors and general health. In patients who present with a longer disease-free interval (beyond 24 months), we would be more inclined to proceed directly to surgery, as demonstrated in this article.

In summary, approximately $2 \%$ of patients with pancreatic cancer who undergo resection (1 out of 250 newly diagnosed patients) could end up being referred to a thoracic surgeon for the management of a pulmonary lesion. They absolutely should be considered to have a unique biology that may benefit from a surgical resection with the intent to cure. The results in these highly selected patients are comparable to those in patients with other solid tumor metastases to the lung. Moreover, a detailed review of the pathology will reveal that nearly $40 \%$ of these patients undergoing resection for a suspected pulmonary metastasis in fact have a second primary lung cancer instead.

\section{References}

1. Katz MH, Wang H, Fleming JB, Sun CC, Hwang RF, Wolff RA, et al. Long-term survival after multidisciplinary management of resected pancreatic adenocarcinoma. Ann Surg Oncol. 2009;16:836-47.

2. Robinson LA, Tanvetyanon T, Springett G, Fontaine J, Toloza E, Hodul P, et al. Pulmonary metastasectomy for suspected pancreaticobiliary cancer. J Thorac Cardiovasc Surg. 2016;152:75-82

3. Downs-Canner S, Zenati M, Boone BA, Varley PR, Steve J, Hogg ME, et al. The indolent nature of pulmonary metastases from ductal adenocarcinoma of the pancreas. J Surg Oncol. 2015;112:80-5.

4. Poruk KE, Kim Y, Cameron JL, He J, Eckhauser FE, Rezaee N, et al. What is the significance of indeterminate pulmonary nodules in patients undergoing resection for pancreatic adenocarcinoma? J Gastrointest Surg. 2015;19:841-7.

5. Thomas RM, Truty MJ, Noqueras-Gonzalez GM, Fleming JB, Vauthey JN, Pisters PW, et al. Selective reoperation for locally recurrent or metastatic pancreatic ductal adenocarcinoma following primary pancreatic resection. J Gastrointest Surg. 2012;16:1696-704.

6. Herman JM, Hoffman JP, Thayer SP, Wolff RA. Management of the primary tumor and limited metastases in patients with metastatic pancreatic cancer. J Natl Compr Canc Netw. 2015;13:e29-36. 